\title{
Therapeutic targeting of mutated p53 in acute lymphoblastic leukemia
}

\section{Frank N. van Leeuwen}

Princess Máxima Center for Pediatric Oncology, Utrecht, the Netherlands

E-mail: FRANK N.VAN LEEUWEN - F.N.vanleeuwen@prinsesmaximacentrum.nl

doi:10.3324/haematol.2019.234872

$\mathrm{I}$ this issue of Haematologica, Demir and colleagues describe a potential novel therapy for TP53-mutated acute lymphoblastic leukemia (ALL). ${ }^{1}$ TP53, the gene encoding p53, is probably the most studied tumor suppressor in cancer biology. In about $50 \%$ of all human cancers, a mutation or deletion of TP53 is observed at some point during tumor development or progression. The high incidence of TP53 loss of function across human tumors relates to its central role as a transcription factor controlling cell cycle regulation in response to DNA damage, DNA repair, metabolic regulation, stem cell development and cell senescence. ${ }^{2}$ In most hematologic malignancies, loss of p53 function is a harbinger of a poor response to therapy, particularly at relapse. ${ }^{3}$ In ALL, genetic alterations affecting TP53 are surprisingly uncommon at diagnosis, with their incidence being less than $5 \% .^{4}$ A notable exception is a rare ALL subtype known as low hypodiploid ALL (32-39 chromosomes), in which there are almost invariably mutations or deletions affecting TP53. In about $50 \%$ of patients with low hypodiploid ALL, TP53 alterations are also present in somatic cells, suggesting that Li-Fraumeni syndrome, an autosomal dominant disorder involving loss of TP53, somehow favors the development of low hypodiploid ALL in children. $^{4}$

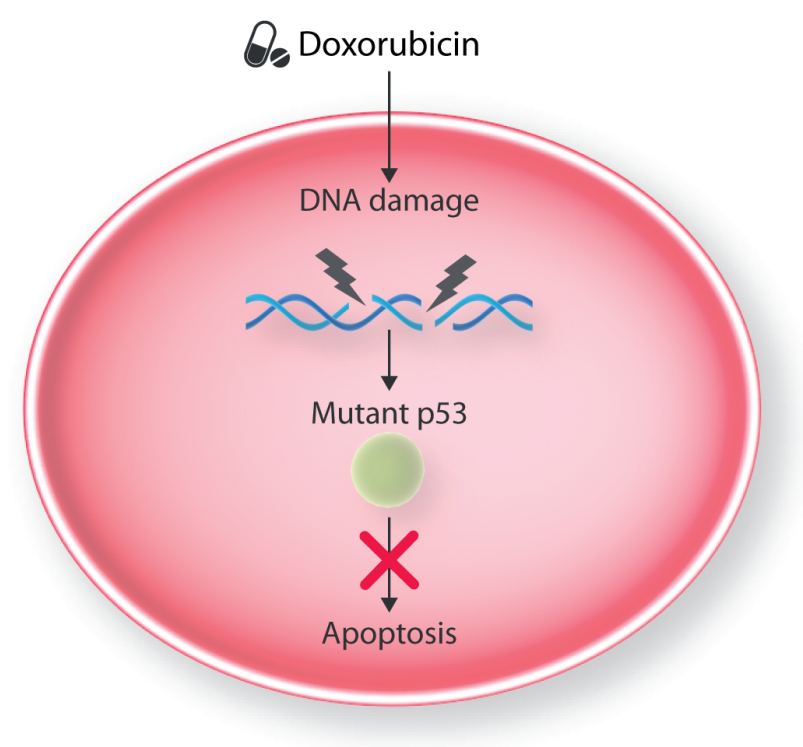

In relapsed ALL, the frequency of TP53 mutations or deletions rises to about $10 \%$ and represents a strong and independent predictor of treatment failure. ${ }^{5}$ TP53 alterations result in either a loss of protein expression or the generation of protein variants with (partly) impaired function. About $80 \%$ of these TP53 variants are the result of missense mutations that affect DNA binding. ${ }^{3}$ In most cancers, the TP53 gene behaves as a classical tumor suppressor, which means that after mutation of one allele, the second allele is lost at a later stage of the disease. However, such a biallelic loss of function is not always observed in ALL, suggesting that dominant-negative effects or haploinsufficiency contribute to leukemogenesis or resistance to therapy. ${ }^{5}$ Regardless of whether a wildtype allele is still present, TP53 mutations or deletions in relapsed ALL predict a highly unfavorable response to therapy. Consequently, the International study for treatment of childhood relapsed ALL (IntReALL), a pan-European study group, now recognizes TP53-aberrant ALL relapse as a rare but very high-risk subtype that requires international collaboration in order to design and test novel treatment protocols, which may include drugs targeting p53.

In the absence of cellular stress signals, expression of the p53 protein is strictly controlled by MDM2 and its

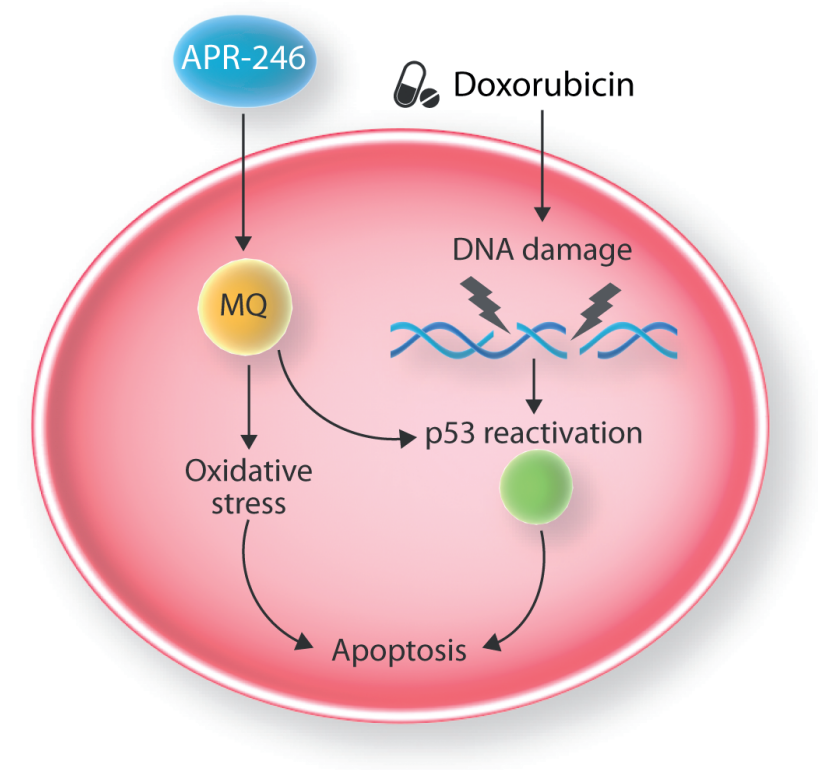

Figure 1. Proposed mechanism of action of APR-246 in TP53-mutated acute lymphoblastic leukemia. Acute lymphoblastic leukemia (ALL) cells expressing mutant p53 are refractory to doxorubicin-induced apoptosis. APR-246 is taken up by cells and metabolized into the reactive electrophile methylene quinuclidinone (MQ). By refolding p53 into an active conformation, MQ restores the apoptosis-inducing effects of doxorubicin. In addition, an increase in oxidative stress brought about by MQ may further enhance apoptosis induction. 
homolog MDMX, which together form an E3 ubiquitin ligase complex that targets $\mathrm{p} 53$, resulting in nuclear export and subsequent degradation. Small molecule antagonists of MDM2 such as nutlins and related compounds, are under clinical evaluation in a variety of cancers expressing wildtype p53. ${ }^{6}$ However, these drugs are ineffective in TP53-mutated or -deleted tumors. To target mutant p53, small molecules have been developed that are predicted to correct p53 protein folding, and thereby restore activity, which would re-enable TP53-mutated tumor cells to undergo apoptosis in response to (chemo)therapy. For instance, cell-based screening of compound libraries has led to the identification of the alkylating compound "p53 reactivation and induction of massive apoptosis" (PRIMA-1) and its methylated derivative PRIMA-1 ${ }^{\text {Met, }}$ also known as APR-246. When taken up by cells, APR-246 is converted to the reactive electrophile methylene quinuclidinone (MQ), which is thought to reactivate conformationally unstable p53 mutants by binding to critical cysteine residues in the p53 DNA binding domain.' Consistent with such a mechanism of action, expression of p53 target genes is restored by these drugs while strongly promoting the induction of apoptosis. However, since the first descriptions of the proposed mechanism of action of this drug, 7,8 alternative mechanisms of action have been put forward. For instance, it was shown that APR-246 induces apoptosis by increasing intracellular levels of reactive oxygen species (ROS), even in fully p53-deficient cells, a mechanism involving glutathione depletion and MQ-mediated inactivation of the enzyme thioredoxin reductase. ${ }^{9}$ Tumor cells expressing only wildtype p53 would be less responsive to these effects, due to the protective role of p53 against oxidative stress. ${ }^{10}$ Therefore, irrespective of its precise mechanism of action, APR-246 appears to selectively target cancers with deficient p53 and for this reason it is under clinical evaluation in a number of TP53-mutated cancers, including myeloid malignancies such as myelodysplastic syndromes and acute myeloid leukemia.

The paper by Demir and colleagues in this issue of the journal describes the potential clinical use of APR-246 in pediatric TP53-mutated ALL. ${ }^{1}$ Using both established ALL cell line models and patient-derived xenografts, the authors compared the effects of this drug in wildtype vs. TP53mutated cells. They observed that the presence of TP53 mutations selectively enhanced resistance to the DNA damaging drug doxorubicin, while increasing sensitivity to APR-246. The induction of apoptosis, seen in TP53-mutated ALL in response to APR-246, was directly correlated with the upregulation of p53 target genes such as $p 21$, $P U M A$ and NOXA, which is at least consistent with pP53 reactivation contributing to these effects. Moreover, when mutant TP53 expression was reduced by shRNA-mediated knockdown, the apoptosis-inducing effects of APR-246 were no longer seen, indicating that in these leukemia models, the response to this drug requires the presence of mutant p53. Given the uncertainties pertaining to the precise mechanism of action of APR-246, the authors examined to what extent intracellular ROS levels may contribute to APR-246-induced apoptosis. Indeed, neutraliza- tion of ROS using MnTBAP, a synthetic metalloporphyrin with antioxidant activity, was able to partially inhibit the apoptosis-inducing activity of APR-246, suggesting a more complex mode of action for this compound than simply restoring p53 target gene expression.

Since doxorubicin is part of the armamentarium used to treat (relapsed) ALL, in vivo synergy between doxorubicin and APR-246 was examined using a TP53-mutated ALL xenograft model. Whereas doxorubicin was ineffective as a single agent in prolonging survival, a clear therapeutic benefit was seen from the treatment with APR-246 alone. In addition, and consistent with the observed ex-vivo synergy between doxorubicin and APR-246, this drug combination further extended leukemia-free survival in the mice studied. Taken together, the work by Demir and colleagues suggests that ALL patients suffering from relapsed TP53-mutated ALL could benefit from the use of APR-246 or similar p53-refolding agents, when used in addition to the current chemotherapy protocol. However, clinical implementation will require a more rigorous characterization of resistance profiles and therapy responses in TP53-mutated ALL, such as the inclusion of more patientderived xenografts and examining potential interactions between APR-246 and other components of the treatment regimen for relapsed ALL. With respect to potential toxicities related to the use of APR-246, it will be important to follow the results of ongoing (phase II/III) trials in acute myeloid leukemia/myelodysplastic syndrome with this drug, either as a single agent or in combination with azacitidine. ${ }^{11}$

\section{References}

1. Demir S, Boldrin E, Sun Q, et al. Therapeutic targeting of mutant p53 in pediatric acute lymphoblastic leukemia. Haematologica 2020; 105(1):170-181

2. Kruiswijk F, Labuschagne CF, Vousden KH. p53 in survival, death and metabolic health: a lifeguard with a licence to kill. Nat Rev Mol Cell Biol. 2015;16(7):393-405.

3. Stengel A, Kern W, Haferlach T, Meggendorfer M, Fasan A, Haferlach C. The impact of TP53 mutations and TP53 deletions on survival varies between AML, ALL, MDS and CLL: an analysis of 3307 cases. Leukemia. 2017;31(3):705-711

4. Comeaux EQ, Mullighan CG. TP53 mutations in hypodiploid acute lymphoblastic leukemia. Cold Spring Harb Perspect Med. 2017;7(3).

5. Hof J, Krentz S, van Schewick C, et al. Mutations and deletions of the TP53 gene predict nonresponse to treatment and poor outcome in first relapse of childhood acute lymphoblastic leukemia. J Clin Oncol. 2011;29(23):3185-3193

6. Burgess A, Chia KM, Haupt S, Thomas D, Haupt Y, Lim E. Clinical overview of MDM2/X-targeted therapies. Front Oncol. 2016;6:7.

7. Lambert JM, Gorzov P, Veprintsev DB, et al. PRIMA-1 reactivates mutant p53 by covalent binding to the core domain. Cancer Cell. 2009;15(5):376-388.

8. Bykov VJ, Issaeva N, Shilov A, et al. Restoration of the tumor suppressor function to mutant p53 by a low-molecular-weight compound. Nat Med. 2002;8(3):282-288.

9. Peng X, Zhang MQ, Conserva F, et al. APR-246/PRIMA-1(MET) inhibits thioredoxin reductase 1 and converts the enzyme to a dedicated NADPH oxidase. Cell Death Dis. 2017;8(4):e2751.

10. Sablina AA, Budanov AV, Ilyinskaya GV, Agapova LS, Kravchenko JE, Chumakov PM. The antioxidant function of the p53 tumor suppressor. Nat Med. 2005;11(12):1306-1313.

11. ClinicalTrials.gov [Internet]. Bethesda (MD): National Library of Medicine (US). Available from: http://clinicaltrials.gov/ct/show/ NCT00287391/NCT03931291/ NCT03745716 